\title{
NECESIDADES SOCIOEDUCATIVAS DE LAS FAMILIAS ANTE EL ABANDONO ESCOLAR PREMATURO
}

\author{
Lucía Álvarez-Blanco \\ Universidad de Oviedo \\ alvarezblucia@uniovi.es \\ Verónica García Díaz \\ Universidad de Oviedo \\ Ellián Tuero \\ Universidad de Oviedo
}

Recepción Artículo: 1 marzo 2020

Admisión Evaluación: 4 marzo 2020

Informe Evaluador 1: 8 abril 2020

Informe Evaluador 2: 22 marzo 2020

Aprobación Publicación: 20 abril 2020

\section{RESUMEN}

Las elevadas tasas de abandono escolar a nivel europeo se erigen como una seria problemática cuyos efectos se originan en el plano individual (fracaso escolar, absentismo, escasa o nula motivación ante el estudio, dificultades de aprendizaje, repetición de curso, baja autoestima, problemas comportamentales y de relación o comunicación con el profesorado y la familia) y se extienden a la esfera de la ciudadanía, viéndose condicionados en consecuencia el capital social y humano. Ante estos episodios de desenganche prematuro de la educación formal, se recuerda y reivindica la urgente cooperación entre las familias y los centros escolares en tanto que agentes de socialización cuyas responsabilidades, influencias mutuas y esfuerzos compartidos responden a unos objetivos comunes de aprendizaje y desarrollo del alumnado/hijo. El trabajo busca conocer las necesidades que manifiestan las familias al implicarse en el aprendizaje de sus hijos a partir de las cuales emerjan indicadores de prevención o protección ante episodios de abandono escolar. Mediante una metodología descriptiva y ex post facto se administró, a una muestra de 140 familias de nacionalidad chipriota, austriaca y española, un cuestionario diseñado ad hoc para evaluar 1) la implicación familiar en las actividades de aprendizaje de sus hijos; 2) la percepción sobre la labor docente y 3) los contactos entre la familia y el centro. Sobresale el reconocimiento parental de la valía profesional, la preocupación hacia la educación y aprendizaje de los hijos adolescentes, así como el interés de asesorarse sobre técnicas de estudio, competencias emocionales y de comunicación a aplicar en el hogar 0 en los escasos, y en la mayoría de las ocasiones insatisfactorios, encuentros con el profesorado. Se concluye reivindicando acciones que, respetando la individualidad de cada familia y alumno, persigan el desarrollo integral y una adaptación social y escolar óptimas.

Palabras clave: implicación educativa familiar; abandono escolar prematuro; adolescencia 


\section{ABSTRACT}

Social and educational needs of families facing early dropout. The high rates of dropout in Europe are considered a severe problem whose effects arise at an individual level (school failure, absenteeism, little or lack of motivation for their studies, learning disabilities, grade repetition, low self-esteem, behavioral and relationship or communication problems with the teachers and the family) and extend to citizenship, shaping hence the social and human capital. Facing this situation of early disengagement of formal education, it should be stressed the urgent cooperation between families and schools as agents of socialization, whose responsibilities, mutual influence, and shared efforts aim to enhance the children's learning and development. This research expects to know some of the necessities of families when they get involved in their children's learning and identify whether these necessities may be used to set prevention or protection indicators in dropout events. The authors, based on a descriptive and ex post facto methodology, used an ad hoc questionnaire among a sample of 140 families from Cyprus, Austria and Spain to assess 1) the family involvement in their children's learning activities; 2) the perception of the teaching practice; and 3) the relationship between the families and the school. Special mention should be made to the parental acknowledgment of the professional skills, the interest in their teenage children's education and learning process, the interest in seeking advice on study techniques, and emotional and communication skills to be applied at home or during the scarce and generally unsatisfactory meetings with teachers. It concludes by demanding actions that strive to full development and an optimal social and school adjustment, always respecting the individuality of each family and student.

Keywords: family partnership; dropping out; adolescence

\section{INTRODUCCIÓN}

Las elevadas tasas de abandono escolar a nivel europeo (Fernández-Lasarte, Goñi, Camino y Ramos-Díaz, 2019; Rodríguez y Guzmán, 2019) se erigen como una seria problemática cuyos efectos se originan en el plano individual -fracaso escolar, absentismo, escasa o nula motivación ante el estudio, dificultades de aprendizaje, repetición de curso, baja autoestima, problemas comportamentales y de relación o comunicación con el profesorado y la familia- (Estévez y Musitu, 2016; Grau, Pina y Sancho, 2011; Méndez y Cerezo, 2018; Urbano y Álvarez, 2019) y se extienden a la esfera de la ciudadanía, viéndose condicionados en consecuencia el capital social y humano (Rogero, 2014), cuestiones estas a las que urge atender por contribuir al desarrollo de potenciales ciudadanos productivos que manifiesten un desarrollo integral, de convivencia positiva y compromiso sociocomunitario (Zubillaga y Gutiérrez, 2018).

Ante estos episodios de desenganche prematuro de la educación formal, se recuerda y reivindica la urgente cooperación entre las familias y los centros escolares en tanto que agentes de socialización cuyas responsabilidades, influencias mutuas y esfuerzos compartidos responden a unos objetivos comunes de aprendizaje y desarrollo del alumnado/hijo (Álvarez-Blanco y Martínez-González, 2016). En nuestra sociedad occidental se advierte la expectativa social de que cada estudiante obtenga un nivel educativo lo más elevado posible, procurando en cualquier caso garantizar la obtención del título de Graduado en E.S.0. o equivalente a nivel internacional. A este respecto, son numerosas las investigaciones que han constatado que para alcanzar esta meta deben concurrir circunstancias como las que siguen: motivación, interés por el estudio/aprendizaje, esfuerzo en la realización de las tareas escolares, adecuadas expectativas académicas y personales, participación en las dinámicas de clase, etc. (Sánchez, 2016).

En contraposición, y revisando alguno de los factores predictores del fracaso y/o abandono prematuro de los estudios, se enuncian entre otros la repetición de curso, las inadecuadas interacciones con los compañeros e integración escolar, el bajo ajuste emocional del estudiante con especial atención a su autoestima (Urbano, Álvarez e Iglesias, 2018), la existencia de problemas comportamentales de diversa importancia y/o el absentismo escolar (Estrada, 2017).

Con ello, y tomando como marco de referencia esta relación de factores, parece conveniente reflexionar sobre el momento de aparición incipiente de dichos fenómenos (García, Casal, Merino y Sánchez, 2013) así como sobre 
el papel que pueden desempeñar en el mismo tanto los padres y madres de este alumnado como el propio colectivo docente. En referencia al origen del fracaso escolar (obtención de bajas calificaciones reiteradas y/o repetición de curso), la investigación apunta hacia los últimos años de la Educación Primaria, circunstancia que debería reforzar las intervenciones que se despliegan desde la institución académica en los cursos previos al acceso a la etapa de Educación Secundaria. Este hecho adquiere aún mayor relevancia al considerar la etapa evolutiva en que se está focalizando: preadolescencia/adolescencia, fase del desarrollo caracterizado por una sucesión de notables transformaciones en la esfera biológico-física, emocional, social, etc. En este punto resulta de interés traer a colación algunos hallazgos de la investigación realizada por Morentín y Ballesteros (2018) mediante 13 historias de vida con estudiantes que no concluyeron con éxito sus estudios de E.S.O., Bachillerato o Formación Profesional Media. Los resultados informan de una paulatina y marcada desvinculación educativa y emocional en el cambio de la enseñanza Primaria a la Secundaria, relevante conclusión ya advertida en trabajos previos de Monarca, Rappoport y Sandoval (2013).

Por su parte, y en referencia expresa al protagonismo que puede ostentar la familia y el profesorado parece aconsejable reforzar la cooperación entre ambos agentes de socialización (Rivas y Ugarte, 2014), atendiendo especialmente al ambiente y dinámica familiar (nivel socioeducativo y cultural de los progenitores, clase social, dinámicas convivenciales, expectativas académicas y personales, implicación educativa parental...) y a las condiciones escolares -actitud y metodologías docente, disponibilidad de recursos, clima comunicativo y de coexistencia...- (Tarabini, 2017). Esta colaboración y alianza es, conforme la literatura científica revisada, un claro indicador de calidad educativa (Parody, Santos, Alcalá y Esequilla, 2019).

En definitiva, y conforme las consideraciones expuestas hasta el momento, parece interesante conocer desde la perspectiva parental algunas necesidades socioeducativas que vivencian al enfrentarse en su día a día a retos asociados con la educación y el aprendizaje de sus hijos adolescentes en riesgo de abandono escolar.

\section{OBJETIVOS}

El objetivo es conocer las necesidades socioeducativas que experimentan las familias con adolescentes en riesgo de abandono escolar. Este diagnóstico girará en torno a dos áreas específicas que la literatura cientííca ha apuntada como relevantes: 1) la implicación familiar en las actividades de aprendizaje de los hijos y 2) la percepción parental sobre la labor del profesorado que imparte clase a estos menores. A resultas de esta detección de necesidades se espera también enunciar sugerencias que contribuyan, por un lado, a una mejora de la comunicación y cooperación entre estos agentes de socialización (familia-centro) y, por otro, que ofrezcan pautas y asesoramiento a las familias sobre el desarrollo positivo de su rol.

\section{MÉTODO}

\section{Participantes}

Contextualizado en el Proyecto Europeo D00R en el que se investigó el abandono escolar prematuro desde una perspectiva comparativa entre el colectivo docente, alumnado adolescente y sus respectivas familias, la muestra es de 140 padres, madres o tutores legales de nacionalidad chipriota ( $n=50 \%)$, española $(n=43.6 \%)$ y austriaca (6.4\%). De estos, el $75 \%$ son madres y el $14.3 \%$ padres, respondiendo un $7.5 \%$ de los progenitores de manera conjunta al cuestionario.

Por otro lado, las franjas de edad que aglutinan a la inmensa mayoría de las familias participantes son las de 31-40 años (45.9\%) y la de 41-50 años (42.1\%). En referencia a los niveles de estudios de los padres se distribuyen como sigue: Estudios Primarios (32.1\%), Formación Profesional (26.4\%), Bachillerato (8.6\%) y Universitarios (3.6\%). En el caso de las madres se corrobora que el 37.1\% tiene estudios Primarios, el 24.3\% de Formación Profesional, el $12.1 \%$ de Bachillerato y tan solo un 1.4\% de Universidad. Por otro lado, y en referencia al estado civil, el $77 \%$ de la muestra está casada, representando el 18\% de las situaciones de separación y divorcio. Por último, la mayoría de las familias tiene dos hijos (65.6\%), el 26.2\% uno y el 8.2\% tres. 


\title{
NECESIDADES SOCIOEDUCATIVAS DE LAS FAMILIAS ANTE EL ABANDONO ESCOLAR PREMATURO
}

\section{Instrumento}

Esta investigación se ha desarrollado a través de una metodología expost- facto, siendo el instrumento de recogida de información utilizado un cuestionario diseñado ad hoc con preguntas de respuesta dicotómica (Sí/No) y cerradas de respuesta tipo Likert conforme la escala: 1-Completamente de acuerdo hasta 5Completamente en desacuerdo (alfa de Cronbach de .8679 para 51 items). Para este trabajo se han seleccionado diversas variables que informan sobre: 1) la implicación familiar en las actividades de aprendizaje de sus hijos y 2) la percepción sobre la labor docente.

\section{Procedimiento}

El Cuestionario de Evaluación de la Implicación Educativa Familiar al que se acaba de aludir en el epígrafe previo fue inicialmente diseñado por Christenson, Lam y Sinclair, y adaptado en posteriores investigaciones por Martínez (1994). No obstante, la versión final, con 51 variables, resultó de una readaptación atendiendo a los requerimientos de la investigación y a la idiosincrasia de las muestras europeas. Por su parte, cada centro confeccionó un listado en que se incluían familias que, teniendo hijos adolescentes en la etapa de E.S.0., cumpliesen Ios requisitos descritos en la segunda nota a pie. Específicamente, en el caso español, y una vez seleccionadas las familias, el jefe de estudios, el profesorado o bien los conserjes contactaron telefónicamente con estas para explicarles los objetivos de la investigación y concertar la realización de la entrevista. En un intento por adaptarse a las necesidades familiares, los investigadores se desplazaron hasta cada instituto en horario de mañana y/0 tarde. Las entrevistas, con una duración aproximada de entre 60-90 minutos, se efectuaron individualmente. Sin embargo, en los restantes países se hizo llegar el cuestionario (base del protocolo de la entrevista) a las familias a través de sus propios hijos, quienes debían de responsabilizarse posteriormente de devolver el cuestionario cumplimentado al profesorado de sus respectivas aulas.

\section{Análisis de datos}

Mediante el paquete estadístico SPSS v.22 se han realizado análisis cuantitativos de tipo descriptivo (frecuencias y porcentajes), comparativo (calculando el estadístico t de Student para muestras independientes) y análisis predictivos. En el caso específico de las familias españolas también se ha administrado el citado cuestionario bajo la modalidad de una entrevista en profundidad, ejecutándose a posteriori un análisis de contenido con las informaciones recabadas.

\section{RESULTADOS}

Para facilitar la comprensión de los resultados, se ha optado por organizar su descripción en función de las categorías anteriormente descritas.

Implicación familiar en las actividades de aprendizaje de sus hijos

Una de las variables más sobresalientes es el reducido hábito lector de estos adolescentes, pues el $70.2 \%$ de los progenitores corrobora que estos no asisten con regularidad a la biblioteca. Tal práctica se ha ido reduciendo a medida que se eleva la etapa escolar y que tiene repercusiones negativas en el aprendizaje de las asignaturas cursadas.

\begin{abstract}
"A mí me decía que iba a la biblioteca pero luego descubrí que seguramente se iba con las amigas. Aún no lo tengo confirmado, ¿eh? A veces la mandas en casa: "Léeme algo" Que te lea...jOye, léeme eso, que tal...Y le cuesta. Ha perdido el hábito siquiera de lo más esencial lo que puede ser leerte cualquier trocito pequeñito de cualquier cosa pero notas que lee con torpeza" (E7, centro escolar español, madre, divorciada, estudios de Bachillerato, una hija de 16 años).
\end{abstract}

Otra de las variables analizadas informa del desconocimiento familiar sobre cómo ayudar a sus hijos/as con sus actividades de aprendizaje, circunstancia patente entre el $72.1 \%$ de los encuestados. En estos casos, los principales focos de dificultad en este asesoramiento radican en: 1) el mero desconocimiento de las estrategias más idóneas para hacerlo, 2) la escasez o desfase de los contenidos que los padres y madres pose- 
en y los que trabajan sus hijos en los institutos 0 , en algunos casos, 3 ) la falta de entendimiento entre ambos que acaba desencadenando riñas y conflictos indeseados por todos. El siguiente comentario así lo refleja:

"Le ayudaba, ahora ya no. Ahora ya, aunque quiera no puedo tampoco... Lo ayudaba en Primaria, sí, a hacer los deberes y a ayudarle y tal pero ahora ya la ayuda no va a más... Ahora ya, en este momento, no le puedo ayudar aunque quiera" (E4, centro escolar español, madre, casada, estudios de Graduado Escolar, un hijo de 15 años).

Por otro lado, y consultados sobre la ayuda parental prestada en la realización de las actividades de aprendizaje de sus hijos, un $62.6 \%$ de los encuestados ayuda a sus hijos, siendo las madres las que más frecuentemente lo hacen. No obstante, llama la atención que un 34.3\% de las familias declara no implicarse en estas tareas, alegando razones como la falta de tiempo, desconocimiento de los contenidos abordados en clase, incapacidad de entenderse con el menor o simplemente señalando que el estudio es una responsabilidad que no les compete como padres bien por ser responsabilidad exclusiva del menor o del profesorado. Sirva de ejemplo la siguiente verbalización:

"No, porque yo no puedo (ayudarle) porque no tengo tiempo. Yo lo ayudaré en lo que sea, que no deje el instituto y eso... Que siga por lo menos un año más hasta los dieciséis. Eso. Ayudarle eso no puedo porque tienes que estar en la casa y tienes que estar ayudando al marido, tengo tres (hijos) más..." (E11, centro escolar español, madre, soltera, Estudios Primarios, hijo de 14 años).

En esta variable se han obtenido diferencias estadísticamente significativas atendiendo al nivel de estudios materno (Chi cuadrado=24.404; $\mathrm{p}=.018$ ) de modo que considerando la opción de respuesta "completamente de acuerdo", el mayor consenso en la prestación de ayuda en tareas escolares radica en aquellas madres que poseen un nivel de estudios primarios (38.9\%), seguidas por las que han cursado Formación Profesional (30.6\%) y las que poseen estudios universitarios (16.7\% de grado de acuerdo). Las que menos ayuda prestan son quienes carecen del graduado escolar (13.9\%).

Por último, y en consonancia con la necesidad que perciben las familias de adquirir información sobre estrategias y métodos con los que prestar ayuda a sus hijos con las actividades de aprendizaje, los análisis constatan que un $81 \%$ de los progenitores se declara de acuerdo 0 en total acuerdo con respecto a esta cuestión. Este interés queda patente en el siguiente comentario:

"Desde el colegio lo más fácil sería que tuviese unas clases de apoyo con los mismo tutores. Porque a veces le mandas a una clase particular y es compartirla con lo que hacen alli (...). Yo soy el primero dispuesto a pagar esos extras porque pagaba las clases particulares y no sirve para nada (...). Yo lo cojo de la mano y le enseño... Que al final no va a sacar el curso, eso ya lo sé yo pero yo no estoy diciendo que es un burro, que esto, que lo otro... Intento por las buenas... Yo lo que quiero es que estudie, si tengo que dejar de fumar yo para pagarle unas clases particulares, dejo, porque yo con mi hijo lo que haga falta" (E36, centro espaol, Isla de la Deva, padre, separado, Formación Profesional, hiio 16 años).

\section{Percepción sobre la labor docente}

Por la representatividad y significatividad de los hallazgos obtenidos en este apartado se hará solamente mención a una variable, la referente a que la valoración general que hacen las familias del profesorado de sus hijos es muy positiva y agradecida, existiendo un $69.1 \%$ que declara que el profesorado se preocupa por su hijo. De esta consideración, como se observa en la siguiente narración, discrepa el 29\% de los progenitores.

\footnotetext{
"El tutor con el que fui a hablar alli para atrás y hablé con el director y el jefe de estudios, hablé el mismo dia con los dos y eran dos opiniones completamente distintas con que sales del colegio y no sabes a quién creer. Los dos me dijeron dos versiones contrarias completamente: que era un niño malísimo, que era un niño noble y muy cariñoso, lo único que no queria estudiar, entonces: ¿a quién hago caso? Decidí no hacer caso a ninguno. No voy (al centro), nada más cuando el chiquillo falta o por buscar al chiquillo, nada más. Te digo la verdad, no me ocupo" (E30, centro escolar español, madre, casada, estudios de Graduado Escolar, hijo de 15 años).
} 


\section{NECESIDADES SOCIOEDUCATIVAS DE LAS FAMILIAS ANTE EL ABANDONO ESCOLAR PREMATURO}

En este sentido, y específicamente para esta variable, y fruto de los análisis de regresión realizados, la Figura 1 representa el modelo integrado por cuatro ítems predictores que explican el 69.6\% de la varianza de la misma.

Figura 1. Modelo predictor de la variable:

"El/la padre/madre considera que el profesorado se preocupa por su hijo".

Resumen del modelo

\begin{tabular}{|c|c|c|c|c|c|c|c|c|c|}
\hline \multirow[b]{2}{*}{ Modelo } & \multirow[b]{2}{*}{$\mathrm{R}$} & \multirow[b]{2}{*}{$\mathrm{R}$ cuadrado } & \multirow[b]{2}{*}{$\begin{array}{l}\text { R cuadrado } \\
\text { corregida }\end{array}$} & \multirow[b]{2}{*}{$\begin{array}{c}\text { Error típ. de la } \\
\text { estimación }\end{array}$} & \multicolumn{5}{|c|}{ Estadísticos de cambio } \\
\hline & & & & & $\begin{array}{l}\text { Cambio en } \\
\text { R cuadrado }\end{array}$ & Cambio en $\mathrm{F}$ & gl1 & $\mathrm{gl} 2$ & $\begin{array}{c}\text { Sig. del } \\
\text { cambio en F }\end{array}$ \\
\hline 1 &, 714 &, 509 &, 493 &, 600 &, 509 & 31,111 & 1 & 30 &, 000 \\
\hline 2 &, 770 & ,592 & ,564 &, 556 & ,083 & 5,929 & 1 & 29 &, 021 \\
\hline 3 & 810 & ,656 & ,619 &, 520 &, 063 & 5,136 & 1 & 28 & 031 \\
\hline 4 &, $857^{d}$ & ,735 & ,696 & ,465 &, 079 & 8,097 & 1 & 27 &, 008 \\
\hline
\end{tabular}

Como se advierte, la percepción parental de que existe una preocupación docente hacia el alumno parece guardar relación con cuestiones de índole docente y familiar. Sobre el profesorado, se apunta como variable de mayor fuerza explicativa de la preocupación docente (49.3\% de la varianza), la existencia de una actitud de estímulo del docente para que al estudiante le guste aprender e ir a clase, idea compartida por el $51.9 \%$ de las familias. Otra de las variables que se ha detectado, apunta la creencia parental de que el profesorado que se preocupa por los estudiantes es aquel que también les enseña cómo estudiar (afirmación señalada por el 44.3\% de la muestra). Por otro lado, en referencia a la propia familia, en el modelo se observa que los progenitores que creen que el profesorado se preocupa por su hijo son aquellos que declaran sentirse bien recibidos cuando asisten en el centro escolar (89.3\% de grado de acuerdo). A modo de ejemplo se aporta la siguiente reflexión:

“. $\mathrm{si}$, si, si, la tutora que es la que yo conoci. Me parece muy maja la tutora y creo que si... Iiene mi teléjono y me avisa de cosas pequeñas que son tan importantes como las más grandes. Quiero decir: es el hecho de que llamen, se preocupen por tu hijo, de que existe el chaval y va a clase y que tal. En épocas pasadas daba igual el guaje, era un centro muy...muy despreocupado, a nivel personal, no sé si porque eran muchos, porque no daban abasto o no sé..." (E22, centro escolar español, padre, separado, estudios de Graduado Escolar, hijo de 15 años).

Por esta razón, la existencia de esta creencia de una preocupación docente por el alumnado es crucial de cara a futuros contactos entre ambos contextos. Además, la otra variable que aparece en el modelo y que alude a la familia, plantea la existencia de dificultades para ayudar al alumno con sus actividades de aprendizaje debido a la necesidad de cuidar a personas mayores, enfermas, etc. (19.9\% de grado de acuerdo al respecto).

\section{DISCUSIÓN Y CONCLUSIONES}

En este trabajo, se ha realizado una aproximación a los dos agentes responsables de la educación y aprendizaje de los adolescentes que se encuentran en situación de riesgo de abandono escolar. Cabe remarcar primeramente que la mayoría de los padres y madres valora positivamente la labor profesional desarrollada por el colectivo docente (Martínez y Molina, 2017) y especialmente por atender aspectos personales del alumnado. La primera conclusión, compartida por autores como Martínez-Gutiérrez y Niemela (2010), informa que si el progenitor percibe que el profesorado se preocupa por su hijo, especialmente en la esfera personal y más allá del rol de alumno que este asume en el aula, mostrará una actitud y disposición más favorable a cooperar, implicarse en la educación y aprendizaje del menor, a atender las Ilamadas del centro o contactar por iniciativa propia ante problemas comportamentales 0 de rendimiento que detecten en el hogar, etc. A idénticos resultados Ilegaron con anterioridad Martínez y Álvarez (2005) en una investigación sobre la relevancia del contexto familiar ante episodios de fracaso y abandono escolar. 
Otra de las conclusiones informa sobre una clara preocupación y ocupación de los padres y madres para con sus hijos (Gordon y Cui, 2012), vehiculizándolas a través de comportamientos como el diálogo sobre lo acontecido en la jornada escolar, la constante actitud de ánimo ante el estudio y/o el aprendizaje (Vázquez, 2018), así como el hecho de proporcionarles todo el material que precisen para el estudio, acompañarles en su estudio y comentarles de manera insistente la relevancia de que se esfuercen, al menos, por alcanzar el título de Graduado en E.S.0. (Córdoba-Caro, Preciado, Pérez, Carrizosa y Molina, 2011).

En este orden de cosas, la mayor demanda de las familias se centra en la necesidad de recibir información y formación sobre estrategias y técnicas de estudio (Álvarez y Martínez-González, 2017) mediante las que hacer más eficaz y menos tensa o conflictiva esa ayuda prestada, afirmación que emana, primeramente, de desconocer cómo poder hacerlo, respuesta en cierto modo esperable dado el mayoritario nivel de estudios Primarios de los encuestados. Con ello se advierte un desfase de los conocimientos que se exige que el menor aprenda, así como de una necesidad por conocer estrategias eficaces de comunicación e interacción con el adolescente junto a herramientas que hagan más motivador el estudio desarrollado en el domicilio familiar (Álvarez, 2012).

En definitiva, esta investigación pone en relevancia el hecho ya corroborado por recientes estudios en la materia (Juliá-Tarveira, Mata-Romeu y Pelegrí-Viaña, 2016; Parody, Santos, Alcalá y Esequilla, 2019) de que la relación entre el colectivo docente, el centro en general y las familias emerge como un factor clave a estimular desde todas las entidades competentes en materia educativa. Una de las opciones mediante las que hacer esto real puede ser la implementación de programas e iniciativas de cooperación entre ambos espacios convivenciales y de aprendizaje (Álvarez-Blanco, 2019; García-Poole, Byrne y Rodrigo, 2019) que se traduzcan tanto en una interacción más habitual, cercana y recíproca en lo referido a la iniciativa del contacto como un espacio ideal y de confianza en que poder expresar e intercambiar sus inquietudes y problemáticas familiares o educativas con aquellos que comparten un mismo sujeto y objeto: el profesorado y las respectivas instituciones escolares (Gârtu, 2017).

\section{REFERENCIAS BIBLIOGRÁFICAS}

Álvarez, L. (2012). Análisis del riesgo de abandono escolar desde la perspectiva del alumnado, del profesorado y de las familias. Identificación de necesidades educativas. Tesis Doctoral. Oviedo: Universidad de Oviedo.

Álvarez-Blanco, L. (2019). Modelos teóircos de implicación familiar: responsabilidades compartidas entre centros educativos, familias y comunidad. Aula Abierta, 48(1), 19-30.

Álvarez-Blanco, L., y Martínez-González, R. A. (2016). Cooperación entre las familias y los centros escolares como medida preventiva del fracaso y del riesgo de abandono escolar en adolescentes. Revista Latinoamericana de Educación Inclusiva, 10(1), 175-192.

Álvarez, L., \& Martínez-González, R. A. (2017). Review of the Partnership between School and Family: A Shared Responsibility. In J. A. González-Pienda, A. Bernardo, J. C. Nuñez \& C. Rodríguez (Eds.), Factors affecting academic performance (pp.121-140). New York: Nova Science Publishers.

Córdoba-Caro, L., Preciado, V., Pérez, L., Carrizosa, M., y Molina, S. (2011). Determinantes socioculturales: su relación con el rendimiento académico en alumnos de Enseñanza Secundaria Obligatoria. Revista de Investigación Educativa, 29(1), 83-96.

Estévez, E., y Musitu, G. (2016). Intervención Psicoeducativa en el Ámbito Familiar, Social y Comunitario. Madrid: Ediciones Paraninfo.

Estrada, M. M. (2017). Estudio descriptivo sobre el abandono escolar temprano e influjo de variables personales y socioculturales en la Ciudad Autónoma de Melilla. Tesis doctoral. Granada: Universidad de Granada.

Fernández-Lasarte, 0., Goñi, E., Camino, I., \& Ramos-Díaz, E. (2019). Perceived social support and school engagement in secondary students. Revista Española de Pedagogía, 77(272), 123-141. doi:10.22550/REP77-1-2019-06

García, M., Casal, J., Merino, R., y Sánchez, A. (2013). Itinerarios de abandono escolar y transiciones tras la 
Educación Secundaria Obligatoria. Revista de Educación, 361, 65-94. doi:10.4438/1988-592X-RE-2011$361-135$

García-Poole, C., Byrne, S., \& Rodrigo, M. J. (2019). Implementation factors that predict positive outcomes in a community-based intervention program for at-risk adolescents. Psychosocial Intervention, 28, 57-65. doi: https://doi.org/10.5093/pi2019a4

Gârtu, M. L. (2017). The School-Family Educational Partnership. Journal of Pedagogy, 2, 107-123. doi: https://doi.org/10.26755/RevPed/2017.2/107

Gordon, M. S., \& Cui, M. (2012). The Effect of School-Specific Parenting Processes on Academic Achievement in Adolescence and Young Adulthood. Family Relations, 61, 728-741. doi: 10.1111/j.17413729.2012.00733.x

Grau, R., Pina, T., y Sancho, C. (2011). Posibles causas del fracaso escolar y el retorno al sistema educativo. Hekadamos, Revista Educativa Digital, 9, 55-76.

Juliá-Tarveira, R., Mata-Romeu, A., y Pelegrí-Viaña, X. (2016). La participación familia-Escuela. Encuentros y desencuentros. Una visión desde los profesionales externos. Documentos de Trabajo Social, (Ed. Esp.), 135143.

Martínez, R. A. (1994). Familia y educación formal. Implicación de la familia en el proceso de enseñanza-aprendizaje. Premios Nacionales de Investigación e Innovación Educativa. Madrid: Ministerio de Educación y Ciencia.

Martínez, R. A., y Álvarez, L. (2005). Fracaso y abandono escolar en educación secundaria obligatoria: implicación de las familias y de los centros escolares. Aula Abierta, 85, 127-146.

Martínez, E., y Molina, E. (2017). Incidencia de factores académicos en el fracaso escolar. Reflexiones derivadas de la experiencia de profesores jubilados. Profesorado: Revista de Curriculum y Formación del Profesorado, número extraordinario (julio), 191-211.

Martínez-Gutiérrez, B., y Niemela, R. (2010). Formas de participación de las familias y de la comunidad hacia el éxito educativo. Revista Educación y Pedagogía, 22(56), 69-78.

Méndez, I., y Cerezo, F. (2018). La repetición escolar en Educación Secundaria y factores de riesgo asociados. Educación XX1, 21(1), 41-61. doi: 10.5944/educXX1.20172

Monarca, H., Rappoport, S., y Sandoval, M. (2013). La configuración de los procesos de inclusión y exclusión educativa. Una lectura desde la transición entre Educación Primaria y Educación Secundaria. Revista de investigación en Educación, 11(3), 192-206.

Morentín, J., y Ballesteros, B. (2018). Desde fuera de la escuela: una reflexión en torno al aprendizaje a partir de trayectorias de abandono escolar prematuro. REICE. Revista Iberoamericana sobre Calidad, Eficacia y Cambio en Educación, 16(1), 5-20.

Parody, L. M., Santos, M. J., Alcalá, M. J., y Esequilla, E. (2019). El desafío educativo del siglo XXI: Relevancia de la cooperación entre familia y escuela. Espiral: Cuadernos del Profesorado, 12(24), $19-29$.

Rivas, S., y Ugarte, C. (2014). Formación docente y cultura participativa del centro educativo: claves para favorecer la participación familia-escuela. ESE: Estudios sobre Educación, 27, 153-168. doi: 10.15581/004.27.153-168

Rodríguez, D., y Guzmán, R. (2019). Rendimiento académico de adolescentes declarados en situación de riesgo. Revista de Investigación Educativa, 37(1), 147-162. doi: 10.6018/rie.37.1.303391

Rogero, J. (2014). Éxito escolar-éxito social y los excluidos del éxito. Revista Nacional e Internacional de Educación Inclusiva, 7(2), 36-52.

Sánchez, A. (2016). Aspectos académicos y personales que inciden en el abandono escolar temprano en educación secundaria obligatoria. (Tesis doctoral), Universidad Complutense de Madrid. Facultad de Educación. Madrid, España.

Tarabini, A. (2017). La meritocracia en la mente del profesorado: un análisis de los discursos docentes en relación 
al éxito, fracaso y abandono escolar. Revista de la Asociación de Sociología de la Educación, 8(3), 349-360. Urbano, A., y Álvarez, L. (2019). La repetición de curso en la adolescencia: influencia de variables sociofamiliares. Revista Educativa Hekademos, 27, 51-59.

Urbano, A., Álvarez, L., y Iglesias, M. T. (2018). Adaptabilidad y cohesión familiar del alumnado de Educación Secundaria Obligatoria. Aula Abierta, 47(2), 237-244. doi: https://bit.ly/3br7Pc2

Vázquez, R. (2018). (Coord.). Hacia una literacidad del fracaso escolar y del abandono temprano desde las voces de adolescentes y jóvenes: Resistencias, «cicatrices» y destinos. Cádiz: Editorial UCA.

Zubillaga, I., y Gutiérrez, M. I. (2018). Pedagogía de la actitud, fruto de la experiencia docente. Revista Panamericana de Pedagogía, 25, 203-225. 
